\title{
An adaptive turbulence filter for decomposition of organized turbulent flows*
}

\author{
G. J. Brereton and A. Kodal \\ Department of Mechanical Engineering and Applied Mechanics, The University of Michigan, Ann Arbor, \\ Michigan 48109
}

(Received 9 March 1993; accepted 5 January 1994)

\begin{abstract}
A new decomposition has been developed in which turbulent processes in shear flows may be represented as a combination of organized and more random turbulent motions. Each component is modeled as a summation of its characteristic eddies, of strength that varies in time and space as a furiction of the entire process. The contribution of all turbulent eddies of the more random component are estimated with an adaptive turbulence filter, which recognizes this component as the orthogonal partner to organized motion, with a power density spectrum of appropriate shape. The decomposition recovers organized motion from time and space series of data in a physically meaningful way, and can be used to characterize interaction between coherent and more random motions. It also provides an estimate for the turbulence in shear flows that are too complex for a meaningful average motion to be identified.
\end{abstract}

\section{INTRODUCTION}

The desire to examine turbulent variables $f_{i}^{\prime}(\mathbf{x}, t)$ as deviations from a class or ensemble average $\left\langle f_{i}(\mathbf{x}, t)\right\rangle$, governed by equations of motion expressed in the same way, has led to numerous proposals for decomposition of turbulent fields, beginning with that of Osborne Reynolds. ${ }^{1}$ Extensions to this original decomposition include the phase average, which has found usage in flows with wave motion (i.e., the works of Phillips ${ }^{2}$ ) and in nonlinear acoustic flows (see, for example, Beyer ${ }^{3}$ ) as well as in turbulent flows with forced repeated periodic unsteadiness. ${ }^{4}$ Ensemble averages conditioned on the occurrence of specific events, such as quadrant analysis, ${ }^{5}$ have also been proposed. Turbulence has also been studied by more advanced decompositions of time and space series of data into orthogonal modes. Methods such as the Karhunen-Loeve expansion have been used to characterize the most energetic turbulent features of near-wall pipe flows as counterrotating streamwise eddy pairs, ${ }^{6}$ and to identify the most energetic coherent motions of an axisymmetric jet. ${ }^{7}$ Moin and Moser $^{8}$ have also examined the most energetic modes of a Karhunen-Loeve decomposition as the characteristic eddies of turbulent channel flow. Stochastic estimation techniques were used by Adrian and $\mathrm{Moin}^{9}$ to identify the large-scale organized "conditional eddies" present in homogeneous turbulent shear flows, whereas Hussain ${ }^{10}$ has demonstrated how coherent motions may be recognized by measuring the degree of coherence in turbulence structures according to the strength of spatial phase correlations in their vorticity.

Techniques for the characterization of turbulence by decomposition of signals into wavelet basis functions have also undergone recent developments, leading to analysis tools, such as the wavelet packet. ${ }^{11}$ More recently, the idea of a class average has been broadened to include averaging

\footnotetext{
* Originally presented at the Special Symposium in honor of William C. Reynolds 60th birthday, 22-23 March 1993.
}

within delineated regions of phase space, in the hope that more meaningful information on turbulent motions might be retained by this exercise. ${ }^{12}$

The characterizations described above typically rely on some a priori information if they are to yield faithful turbulent decompositions. Reynolds decompositions and quadrant analyses require a knowledge of the stationarity of the mean flow if deviatoric components are to be classified as turbulent. Unsteady-flow phase averages necessitate additional information on the reference positions at which ensembles begin, as well as a guarantee of repeatability in organized motion from ensemble to ensemble. While proper orthogonal decompositions organize the greatest proportion of the energy of turbulence data into the fewest number of modes, additional prescriptions may be necessary to identify modes (singly or grouped) that characterize individual physical structures. In flows that feature nonstationarity in time, organization that lacks repeatability, and inhomogeneity in space with many different structures of comparable energy, which may be termed complex flows, the utility of existing methods for improved understanding of turbulence may be small. In such cases, approximation techniques are of value for estimating the turbulent and organized components of complex flow fields and studying their characteristics.

The approximation techniques described and applied in this paper are based on the premise that projections of the turbulent contribution of time and space series of data have distinctive mean square representations that may be recognized and isolated from those of residual organized (temporally or spatially) motions. This approach is in contrast to the traditional one of treating turbulence as deviatoric from an organized field, which may be identified by a prescribed averaging procedure. It treats complex flows as though the character of turbulent motions may be identified more readily than that of organized ones. The decomposition technique is presented in the form of an adap. tive characteristic eddy function or turbulence filter, together with applications that demonstrate cycle-by-cycle 
decompositions of turbulence in forced unsteady boundary layers and reveal the momentary spatial organization in a turbulent jet.

\section{ORGANIZATION IN TURBULENT FLOW}

There is considerable evidence that turbulent flows have organized features, ${ }^{13-17}$ but no unambiguous means of identification that is of general applicability to all turbulent flows. For example, the numerous techniques for identifying coherent patterns of events in near-wall turbulence do not necessarily detect organized motions in other flowsthe link between the organization and its identification has had to be established by careful study of that particular flow. The effectiveness of identification techniques, and the organized motion they define, depends on the information available. Motions readily detectable in a threedimensional space series may make no more than smeared footprints or kinks in a plane or line through the same domain. Thus, a one-dimensional data series of velocity measurements away from the wall in a boundary layer might give no indication of organization in fluid motion, yet a space series in the $x-y$ plane through the same point might reveal eddying motions of obviously organized form.

The technique described in this paper is a new, general, first-order approximation method that can extract the most significant organized motions revealed by a data series from turbulent shear flows. The technique has no restriction to any class of turbulent flow, providing the data series are well resolved. It defines organized motion as the orthogonal partner to the "background" turbulent part of the data series, which has a power spectrum of the same shape as in stationary turbulent shear flow. Thus motions that (momentarily) cause significant deviation from the expected data-adaptive shape of the background turbulence spectrum are termed organized. These can be the largest naturally organized motions that span entire domains, as in the large-scale coherent structures of jets and wakes, or flow structures that differ from background motion through effects of initial and boundary conditions, or motions that follow external organized forcing. The generality of this approach does not allow distinction between these effects, though other techniques have been developed for these purposes in particular flows. Instead, it gives a firstorder description of the most organized motions made visible by the information provided. The technique is described in terms of a turbulence filter below.

\section{A TURBULENCE FILTER}

The characterization of turbulence with a filter is equivalent to representing turbulence as a sum of many characteristic eddies, whose strength varies in time or space as another process. Lumley ${ }^{18}$ pioneered this idea in the form $u^{\prime}=\phi * g$, where $u^{\prime}$ is a member of an ensemble of characteristic eddies, $g$ represents the strength of eddies as a random process, $\phi$ is the characteristic eddy function, and $*$ is the convolution operator. This approach may be extended to the characterization of complex (unsteady, spatially varying) turbulent flows from which the temporal/spatial mean has been removed, in the form of a decomposition of organized (in space or time) and turbulent components. In the approach described here, the summed effects of organized motion and background turbulence in a data series $u$ are given, respectively, by the decomposition: $u=\widetilde{u}+u^{\prime}$.

For a series of measured data $u$ from which the mean has been removed, its turbulent component may be expressed as

$$
u^{\prime}=\phi * u,
$$

if an estimate of the characteristic turbulence function $\phi$ of all turbulent eddies is provided. The nonturbulent or organized part is then

$$
\tilde{u}=u-u^{\prime} \text { or } \tilde{u}=[\delta()-\phi] * u,
$$

where the argument of $\delta$, the Dirac delta function, is either time or position, depending on the kind of series of data. In a time series of velocity data measured in an unsteady turbulent flow, $\tilde{u}(t)$ might represent the organized unsteady motion deviatoric from the long-time mean (such as oscillation); in a space series, such as a velocity field deduced from a planar particle-image-velocimetry measurement, $\tilde{u}(x, y)$ could describe the organized spatial deviation from the spatial mean, such as swirl and inhomogeneity in the mean flow. One may also propose functions $\phi$, which describe subclasses of turbulent eddies with particular attributes, allowing successive decompositions of $u^{\prime}$ into subcomponents. If no organized motion is extracted, one such function for subclasses of turbulent eddies isolates the most energetic mode of a Karhunen-Loeve decompositionmotions described by this mode are commonly known as those of the characteristic eddy.

Since complete information is unavailable to guide the choice of which parts of a data series in a complex flow are locally turbulent and that are not, the determination of the characteristic turbulence function is posed as an estimation problem. Considering, for simplicity, a time series of data $u(t)$, we wish to choose a function $\phi(t)$ that minimizes the discrepancy between $u^{\prime}(t)$ and $u_{\text {est }}^{\prime}(t)$, where

$$
\begin{aligned}
& u_{\text {est }}^{\prime}(t)=\phi(t) * u(t), \quad \text { in which case } \\
& \tilde{u}_{\text {est }}(t)=[\delta(t)-\phi(t)] * u(t) .
\end{aligned}
$$

Since $u_{\mathrm{est}}^{\prime}(t)$ and $u(t)$ must be real, $\phi(t)$ must be a real function, though its Fourier transform $\Phi(f)$ may, in general, be complex.

It is convenient for the decomposed components to be orthogonal, in the sense that

$$
\overline{\widetilde{u}_{\text {est }}(t) u_{\text {est }}^{\prime}(t+\tau)}=0, \quad \text { at } \quad \tau=0,
$$

where the overbar denotes an average over all $t$. Under this condition, the triple-decomposed forms of the NavierStokes equations (Hussain and Reynolds ${ }^{4}$ ) apply directly to the decomposed variables. The constraint that the summed energy of the decomposed quantities cannot exceed that of the total data series at any frequency requires the filter $\phi(t)$ to be bounded, in the frequency domain, as 
$0<|\Phi(f)|<1$.

Suppose the true value of the turbulent component were known a priori (for example, as measured in a flow with no mean, such as decaying isotropic turbulence in a box). An estimate of the turbulent component using (3) would be considered optimal if the mean square error $E$ between the estimated and the true turbulent components could be minimized. By writing the filtered decomposition of (3) in the Fourier domain as

$$
U_{\text {est }}^{\prime}(f)=\Phi(f) U(f),
$$

and using Rayleigh's theorem, the minimization of the mean square error $E$ is expressed as

$$
E=\frac{1}{2 \pi} \int_{-\infty}^{\infty}\left|U_{\mathrm{est}}^{\prime}(f)-U^{\prime}(f)\right|^{2} d f=\text { minimum }
$$

Since $U_{\text {est }}^{\prime}(f)=\Phi(f) U(f)$, then

$$
\begin{aligned}
E & =\frac{1}{2 \pi} \int_{-\infty}^{\infty}\left|\Phi(f) U(f)-U^{\prime}(f)\right|^{2} d f \\
& =\text { minimum. }
\end{aligned}
$$

If $u^{\prime}(t)$ and $u(t)$ are jointly wide-sense stationary processes and have zero means, then the optimum filter function that minimizes this integral can be found (see, for example, Peebles ${ }^{19}$ ) as

$$
\Phi_{\mathrm{opt}}(f)=\frac{S_{u u^{\prime}}(f)}{S_{u u}(f)}, \quad \text { with } E=\frac{1}{2 \pi} \int_{-\infty}^{\infty} \frac{S_{u^{\prime} u^{\prime}}(f) S_{u u}(f)-\left|S_{u u^{\prime}}(f)\right|^{2}}{S_{u u}(f)} d f
$$

Here $S_{u u}(f)$ is the energy density spectrum of $u(t)$ and $S_{u u^{\prime}}(f)$ is the energy density cross-spectrum of $u(t)$ and $u^{\prime}(t)$. In general, $\Phi_{\text {opt }}(f)$ and $S_{u u^{\prime}}(f)$ are complex. One can reexpress the filter of $(9)$ in the form

$$
\Phi_{\mathrm{opt}}(f)=\left|\Phi_{\mathrm{opt}}(f)\right| e^{i<\Phi_{\mathrm{opt}}(f)},
$$

where the modulus of this optimal filter is

$$
\left|\Phi_{\mathrm{opt}}(f)\right|=\left(\frac{S_{u^{\prime} u^{\prime}}(f)}{S_{u u}(f)}\right)^{1 / 2}\left(\frac{S_{u^{\prime} u^{\prime}}(f)+S_{\widetilde{u u}}(f)}{S_{u u}(f)}\right)^{1 / 2},
$$

and its phase is

$$
\angle \Phi_{\mathrm{opt}}(f)=\arctan \frac{\operatorname{Im}\left[U^{\prime}(f) \tilde{U}^{*}(f)\right]}{\operatorname{Re}\left[U^{\prime}(f) \tilde{U}^{*}(f)\right]+\left|U^{\prime}(f)\right|^{2}},
$$

where * denotes a complex conjugate. To specify this optimal filter, estimates of $S_{u^{\prime} u^{\prime}}(f)$ and $S_{u^{\prime} \tilde{u}}(f)$ are required. If the cross-spectral energy density of organized and turbulent motion is assumed to be small, the second term on the right-hand side of (11) approaches unity; if it is an even function, and so equal to its cospectrum, the filter phase is zero. Under these assumptions, the turbulence filter is then

$$
\Phi(f)=\left(\frac{S_{u^{\prime} u^{\prime}}(f)}{S_{u u}(f)}\right)^{1 / 2} .
$$

Thus the optimal filter for extracting $\tilde{u}$ and $u^{\prime}$ from $u$ can deduced from the measured spectrum $S_{u u}(f)$, and an estimate of the spectrum of the turbulent component $S_{u^{\prime} u^{\prime}}(f)$, with no knowledge of $\tilde{u}$ required. For the example of decaying isotropic turbulence in a box, where $u^{\prime}=u$, $S_{u^{\prime} u^{\prime}}(f)$ would be estimated as $S_{u u}(f)$, so that $\Phi(f)=1$ and a perfect recovery of the true turbulent component would be achieved by the filter. In general, the "true" turbulent component is not known, and so an estimate of
$S_{u^{\prime} u^{\prime}}(f)$ is used to recover estimates of $\tilde{u}$ and $u^{\prime}$ from the measured data series $u$. However, the development of the optimal filter is unchanged and still leads to (9) and hence (13).

Substitution of (13) into (9) and further manipulation allows its mean-square error to be expressed as

$$
\begin{aligned}
E= & \frac{1}{2 \pi} \int_{-\infty}^{\infty} \frac{S_{\widetilde{u}_{\text {est }} \tilde{u}_{\mathrm{est}}}(f) S_{u_{\mathrm{set}}^{\prime} u_{\mathrm{est}}^{\prime}}(f)}{S_{u u}(f)} \\
& -S_{u u}(f)\left[\Phi(f)-\Phi^{2}(f)\right]^{2} d f .
\end{aligned}
$$

It is small when either process is narrow band or at frequencies when the spectral energy density of one component greatly exceeds that of the other. This filter has been shown to be iterative (Brereton and $\mathrm{Kodal}^{20}$ ) in the following sense: when $S_{u u}(f)$ is measured from the data scrics $u(t)$, an estimate of $S_{u^{\prime} u^{\prime}}(f)$ allows recovery of $u_{\mathrm{est}}^{\prime}(t)$ as $\phi(t) * u(t)$, and assures the spectral density of $u_{\text {est }}^{\prime}(t)$ matches its original estimate. Thus, iteration through trial estimates of $S_{u^{\prime} u^{\prime}}(f)$ can guide minimization of functions of the estimated data series $u_{\text {est }}^{\prime}(t)$ and $\tilde{u}_{\text {est }}(t)$.

The adaptive-filter approach to decomposition is then to measure $S_{u u}(f)$ and make an initial estimate of $S_{u^{\prime} u^{\prime}}(f)$ as the part of $S_{u u}(f)$ with the expected shape of a shear-flow turbulence spectrum. One may then iterate on $\Phi(f)$ over all $f$ until $\Phi(f) U(f)$ and $[1-\Phi(f)] U(f)$ yield estimates of $U^{\prime}(f)$ and $\widetilde{U}(f)$, whose time-domain counterparts arc orthogonal, as required by (4), under the realizability constraint (5), and with a smooth background-turbulence spectrum $S_{u^{\prime} u^{\prime}}(f)$ (see Sec. IV B). The departure of $u_{\text {est }}^{\prime}(t) \tilde{u}_{\text {est }}(t)$ from orthogonality is then the quantity that is minimized. Two- and threedimensional space series may be decomposed in the same way using straightforward extensions to these results. Since this filter was deduced from considerations of minimization of the square of the error between the estimate and the "true" value of a component of the data series, the accu- 
racy of estimates is of second order with respect to the accuracy with which the filter is determined.

\section{FILTER IMPLEMENTATION}

\section{A. Estimation of the background turbulence spectrum}

Decomposition by a filter of this kind presupposes that a reasonable initial estimate of the contribution of one of the decomposed fields to the energy spectrum of the data series can be made. While temporally or spatially organized motions in complex flow lack any general spectral prescription, the shapes of turbulent energy spectra in shear flows are well known and distinctive. Even for $\operatorname{Re}_{\lambda} \lesssim 300$ (where $\lambda$ is the Taylor microscale), it is quite usual for energy spectra to feature an extended range of algebraic decay in the form $S(f) \propto f^{-n}$, where $\frac{5}{3}<n<2 .{ }^{21}$ This smoothness may be exploited through spectral descriptions given by simple analytical models. Time-series spectra of single component velocity measurements typically feature a flat low-frequency shape, consistent with the form of one-dimensional von Kármán spectra. The flat low-frequency behavior and decaying high-frequency behavior is also well modeled as a low-order autoregressive process. ${ }^{22}$ In situations in which one has access to all components of the velocity field, it can be useful to approximate turbulent power spectra by models such as the threedimensional von Kármán energy spectrum:

$$
S(k)=\frac{\overline{u_{i}^{\prime} u_{i}^{\prime}}(k L)^{4}}{\left[1+(k L)^{2}\right]^{p}}
$$

(where $p>1, k=\sqrt{k_{x}^{2}+k_{y}^{2}+k_{z}^{2}}$ is the wave number, and $L$ is a characteristic length scale), in which the resolved lowfrequency region of the spectrum tends to zero with decreasing frequency. Smoothly blended versions of the $k^{2} \sim k^{-5 / 3}$ model proposed by Reynolds ${ }^{23}$ for initial fields in turbulence simulations can also approximate measured turbulence spectra well. Integrated forms of these models can be fitted to spectra of individual components of velocity, which frequently share similarities in shape to the spectrum of the total energy. This observation may be made from the results of Rogallo ${ }^{24}$ for computations of homogeneous shear turbulence, and can be inferred from aliased time-series spectra from the data of Perry et al. ${ }^{25}$

Use of the characteristic spectral shapes of stationary shear flows as initial estimates of $S_{u^{\prime} u^{\prime}}$ in complex flows is lent justification by some recent results from studies of periodic unsteady turbulent flow. In these studies, when the organized disturbance was perfectly repeatable from cycle to cycle, and the use of a phase-average decomposition justified, energy spectra of the turbulent component of streamwise velocity were of almost identical shape to energy spectra measured in steady flow under comparable mean conditions. ${ }^{26}$ A series of energy spectra of $u(t)$, measured in turbulent pipe flow at frequencies as high as four times the mean flow burst frequency, are shown in Fig. 1 with the companion decomposed $u^{\prime}(t)$ spectra in Fig. 2 . For the narrow-band sinusoidal forcing of these experiments, ${ }^{27}$ it is clear that there is, on average, no ap-

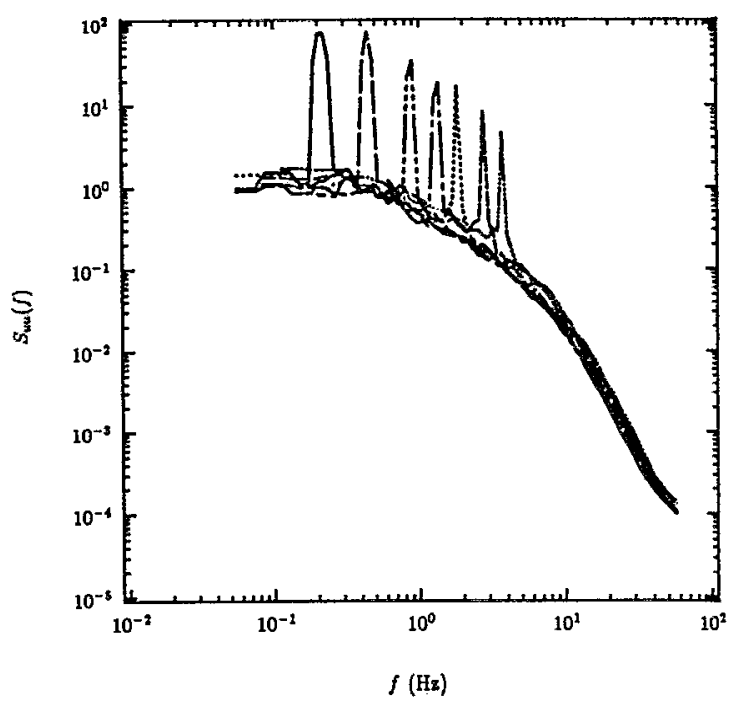

FIG. 1. Energy spectral densities of $u(t)$ measured in turbulent pipe flow $\left(\operatorname{Re}_{D}=12000, y^{+}=75\right)$ undergoing forced sinusoidal oscillation about its mean. The frequency range is from 0.2 to $4 \mathrm{~Hz}$, with a mean flow burst frequency of around $1 \mathrm{~Hz}$.

preciable modification of the energy content of $u^{\prime}$, and so turbulence energy spectra from stationary flows provide useful estimates of turbulence energy spectra in periodic unsteady flows. Further support for the idea that shear flows retain the same general spectral shape when undergoing deformation is provided by the rapid-distortiontheory results of Hunt and Carruthers. ${ }^{21}$ In their calculations of homogeneous shear turbulence, their computed spectra retain the same shape over wide ranges of applied strain. More recent support has been lent by the work of Aubry, ${ }^{28,29}$ who has demonstrated how the related biorthogonal decomposition establishes spectral scaling

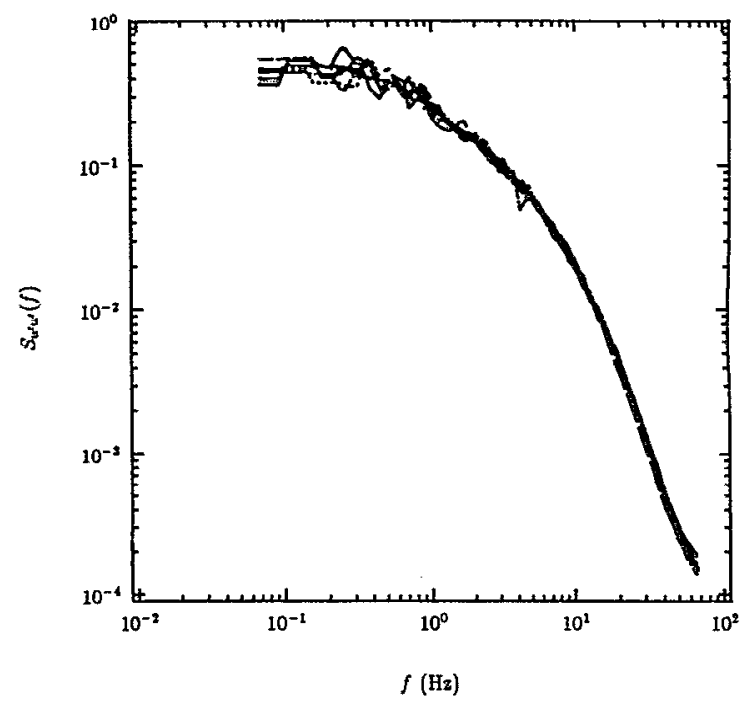

FIG. 2. Energy spectral densities of $u^{\prime}(t)$ deduced from the data of Fig. 1 by phase averaging. Each spectrum is plotted with the same line as in Fig. 1. 
laws for inhomogeneous flows, through dilation symmetries of the turbulent Navier-Stokes equations.

\section{B. Discretization in frequency space}

The procedure for optimizing the turbulence filter is to make an initial estimate of $S_{u^{\prime} u^{\prime}}(f)$, discretized into frequency intervals in logarithmic space, and then iterate on the value of $S_{u^{\prime} u^{\prime}}(f)$ at each interval, and hence the estimates of $\tilde{u}$ and $u^{\prime}$, until $\left|\overline{\tilde{u}}_{\text {est }}(t) u_{\text {est }}^{\prime}(t)\right|$ is minimized. The estimated turbulent contribution to the energy spectrum is chosen as either a low-order autoregressive model or a wave number model, which passes through the extreme frequencies of the energy spectrum of the data series. It is assumed that the data series is of sufficient size and accuracy to allow a converged energy spectrum for $S_{u u}(f)$ to be estimated by standard techniques (i.e., by discrete Fourier transform). It is also assumed that the data sampling frequency and its duration are sufficient to resolve the highest and lowest frequencies in the energy spectrum as turbulent motions.

Discretization of the energy spectrum is necessary to keep the minimization problem to manageable proportions. To assure that the character of the energy spectrum is retained with reasonable fidelity, the maximum frequency interval for discretization is chosen as one in which the energy absorbed by eddies in that interval from all larger scales is expected to be an order of magnitude smaller than the amount of energy transfer to eddies smaller in scale by one frequency interval. In this way, the smoothing of $S_{u^{\prime} u^{\prime}}(f)$ from discretization over each interval is an order of magnitude less significant than the total energy transfer from eddies in that frequency interval, and so should not impair spectral representation of the energy cascade significantly. Isotropic models of energy spectra in the inertial subrange [of the order of the steepest gradients of $S_{u^{\prime} u^{\prime}}(f)$ in typical experiments] may then be coupled with energy cascade arguments (Tennekes and Lumley ${ }^{30}$ ) to estimate the frequency interval over which energy transfer to smaller scales exceeds energy absorption by about ten times. This analysis suggests intervals of central frequency $f$, of bandwidth $\Delta f^{+}+\Delta f^{-}$, equispaced along a logarithmic axis described by

$$
\begin{aligned}
\ln \left(\frac{f+\Delta f^{+}}{f}\right) \simeq & \ln \left(\frac{f}{f-\Delta f^{-}}\right) \simeq \frac{1}{20} \\
& \text { with } \frac{\Delta f^{+}+\Delta f^{-}}{f} \simeq \frac{1}{10} .
\end{aligned}
$$

In addition to discretization, estimates of $S_{u^{\prime} u^{\prime}}(f)$ are replaced by smoothed versions [a piecewise cubic curve fitted through four adjacent values of $\left.S_{u^{\prime} u^{\prime}}(f)\right]$ at each stage of iteration. This constraint imposes additional smoothing, justified by the well-known characterization of steady turbulent shear flows as comprising a continuous range of scales that produce a smooth energy spectrum. Finally, the initial estimate of $S_{u^{\prime} u^{\prime}}(f)$ and the corresponding $\Phi(f)$ are used to bound the minimization at each $f$, such that if $\Phi_{\text {init }}>0.5$ it is constrained to remain above 0.5 , whereas if $\Phi_{\text {init }}<0.5$ it must remain below 0.5 .

\section{Minimization procedure}

The constrained problem of minimizing $\left|\overline{\widetilde{u}_{\text {est }}(t) u_{\text {est }}^{\prime}(t)}\right|$ by variation of $\Phi(f)$ over all values of discretized $f$ space is solved using quasi-Newton or conjugate gradient methods. Dependence of the solution on the initial estimate of $S_{u^{\prime} u^{\prime}}$, and the choice of convergence tolerance for the minimization algorithm is reduced by systematic variation of both (usually over a small range) until the minimum minimorum is achieved. In practical applications, multiple local minima may be found, bringing into question the uniqueness of a decomposition by an approximation technique. Since turbulence is a phenomenon of high dimension, which can be decomposed in many ways into multiple orthogonal modes, then, provided the most energetic ones are resolved within their respective fields, uncertainty over the allocation of a small number of lowenergy modes will have a minimal effect on most statistical measures. For the data series studied to date, the proximity of these minima was so close that statistical descriptions of data decomposed about different local minima were indistinguishable. Thus, the resultant turbulence filter apportions each frequency component of a data series into turbulent and organized parts of the series, guided by an initial estimate of the expected energy spectrum of the turbulence component. In this way, motions more pronounced in amplitude than expected turbulence levels, or of behavior better suited to fits of Fourier expansions than turbulence, will be recognized as organized motion, as the orthogonal partner to turbulent motion.

\section{Nonstationarity and localization}

Since orthogonality between components of decomposed velocity fields is a desirable property (i.e., in Reynolds and triple decompositions, where it is enforced), it is useful to consider how it might be achieved by a filtered decomposition. For a stationary series of real data, the condition that $\overline{\widetilde{u}(t) u^{\prime}(t)}=0$ may be expressed in Fourier space by the power theorem to yield

$$
\int_{-\infty}^{\infty} \tilde{U}^{*}(f) U^{\prime}(f) d f=0 .
$$

If $\widetilde{U}(f)$ and $U^{\prime}(f)$ are replaced by estimates made by a real filter, the orthogonality condition is expressed as

$$
\int_{-\infty}^{\infty}|U(f)|^{2}\left[\Phi(f)-\Phi^{2}(f)\right] d f \simeq 0,
$$

the approximate equality indicating that estimates are now employed. For the case of a stationary data series, orthogonality can only be achieved by a real filter $\Phi(f)$ that is equal to 0 or 1 at any frequency. If the estimated turbulence spectrum is to be continuous, a filter decomposition could only then achieve orthogonality for the degenerate cases of either $u=u^{\prime}$ or $u=\widetilde{u}$. For an inhomogeneous or nonstationary data series, or complex filters, these restrictions on $\Phi(f)$ do not apply. However, when data series 
approach stationarity or homogeneity, orthogonality is likely to be achieved with $\Phi(f)$ close to 0 or 1 . In such cases, the enforced smoothness of the spectrum of the estimated turbulent component $S_{u^{\prime} u^{\prime}}(f)$ (see Sec. IV B) can strongly influence iteration toward the filter, which minimizes the departure from orthogonality.

Localization of spectral estimates in time/space is achieved by time-frequency analysis techniques. Of these, the instantaneous power spectrum ${ }^{31}$ has the desirable properties of an unambiguous definition of a time-varying spectrum and relative insensitivity to nonoptimal choices of a window function that are well suited to this application. When nonstationarity or inhomogeneity is significant, the turbulence filter may be constructed in the form $\Phi(f, t)$ to make a time/space adaptive split of the localized Fourier transform of $u$ into its organized and background turbulent contributions.

\section{APPLICATIONS TO ONE-DIMENSIONAL DATA}

Time series of a single component of velocity may be characterized with a one-dimensional version of the filter described in Sec. III. The data considered were a time series of the streamwise component of velocity measured at a single point in a flat plate turbulent boundary $\left(\operatorname{Re}_{\theta}=3200, y^{+}=400\right)$, with and without forced oscillation of the free stream about its mean. These measurements were made in a water tunnel, in which the RMS freestream disturbance level was measured at approximately $0.2 \%$ of $U_{\infty} \cdot{ }^{32}$ As a test of the fidelity of the filter in distinguishing between turbulent and organized unsteady motions, its performance was considered first in a stationary flat-plate boundary layer.

\section{A. Time series of $u$ in a stationary turbulent flow}

The coherent turbulent motions of the boundary layer arc spatiotemporally complex, and are short-lived in their most organized states ("sudden oscillation, bursting, and ejection" accounting for $80 \%$ of the turbulence production in $20 \%$ of the boundary layer ${ }^{33}$ ). Consequently, they reveal little of their organization in an energy spectra of stationary time series of data measured at a single point. A turbulence filter decomposition should identify almost the entire data series as turbulent. In this test application, a time series of 16384 data was considered, of duration approximately 500 large-eddy time scales, sampled at about twice the estimated Kolmogorov frequency. The frequency extremes of the energy spectrum therefore correspond to small-scale turbulence and aliased large-scale turbulent motion. The energy spectrum $S_{u u}(f)$ was estimated and discretized into 50 adjacent frequency bands following the procedure of Sec. IV. The energy spectrum of a first-order autoregressive process was fitted through the highest and lowest frequencies of the discretized spectrum to serve as $S_{u_{\text {est }}^{\prime} u_{\text {est }}^{\prime}}(f)$, shown in Fig. 3 , together with $S_{u u}(f)$. After applying the constraints and discretization of Sec. IV, the minimization of the cross-correlation of $u^{\prime}(t)$ and $\widetilde{u}(t)$

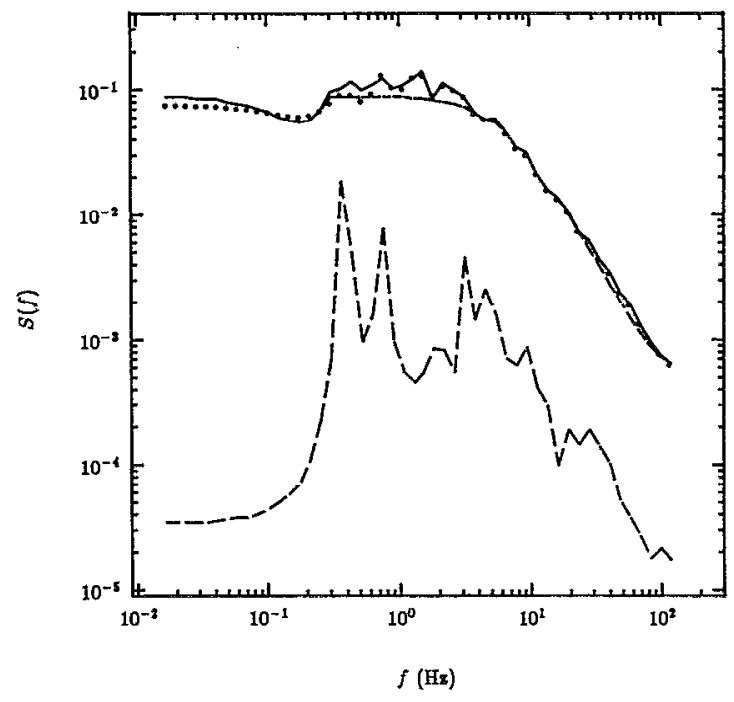

FIG. 3. Measured energy spectral densities of $u(t)$ and estimated energy spectral densities of $\tilde{u}(t)$ and $u^{\prime}(t)$ in a steady flat-plate boundary layer $\left(\operatorname{Re}_{\theta}=3200, y^{+}=400\right)$, according to the turbulence filter decomposition;--, the spectrum of $u(t) ;---$, the initial estimate of the $u^{\prime}(t)$ spectrum; $\cdots$, the decomposed $u^{\prime}(t)$ spectrum;-- , the decomposed $\widetilde{u}(t)$ spectrum.

was carried out in 50 space. For this data set, the optimal filter yielded a cross-correlation coefficient between $\widetilde{u}_{\text {est }}$ and $u_{\text {est }}^{\prime}$ of the order of $10^{-9}$.

The energy spectra of the decomposed data series are also included in Fig. 3. The minimization converges toward $\Phi(f)=1$ over most of the spectrum, identifying the flow as one with little apparent organization, from the perspective of a single-point time series. Statistics of the filtered turbulence field were almost indistinguishable from those of the measured data series. Of particular interest were the peaks in $S_{\widetilde{u}_{\text {est }} \widetilde{u}_{\text {est }}}$, some of which were attributed to (organized) vibration of the LDV measurement system relative to the water tunnel, and had previously passed undetected. The proportion of energy contained in motions estimated to be organized was approximately $2 \%$ of the total; this percentage may be taken as the order of uncertainty that arises through discretization of the spectrum, numerical rounding errors, and enforcement of constraints, which prevent convergence to the degenerate solution: $\Phi(f)=1 ; u(t)=u^{\prime}(t)$. That a cross-correlation coefficient of $10^{-9}$ can be reached with $\Phi(f) \neq 1$ is consistent with the results of other statistical tests, which indicated that this data set did not completely satisfy the requirements of strict stationarity, which are almost impossible to attain in laboratory experiments. An accuracy of about $2 \%$ in energy for an estimation technique of this kind instills confidence in the method and its application.

\section{B. Time series of $\boldsymbol{u}$ in forced unsteady turbulent flow}

A more interesting decomposition problem is that of boundary-layer turbulence subjected to forced sinusoidal oscillation of the free stream about its mean. In the case studied, the forcing period was about eight large-eddy time 


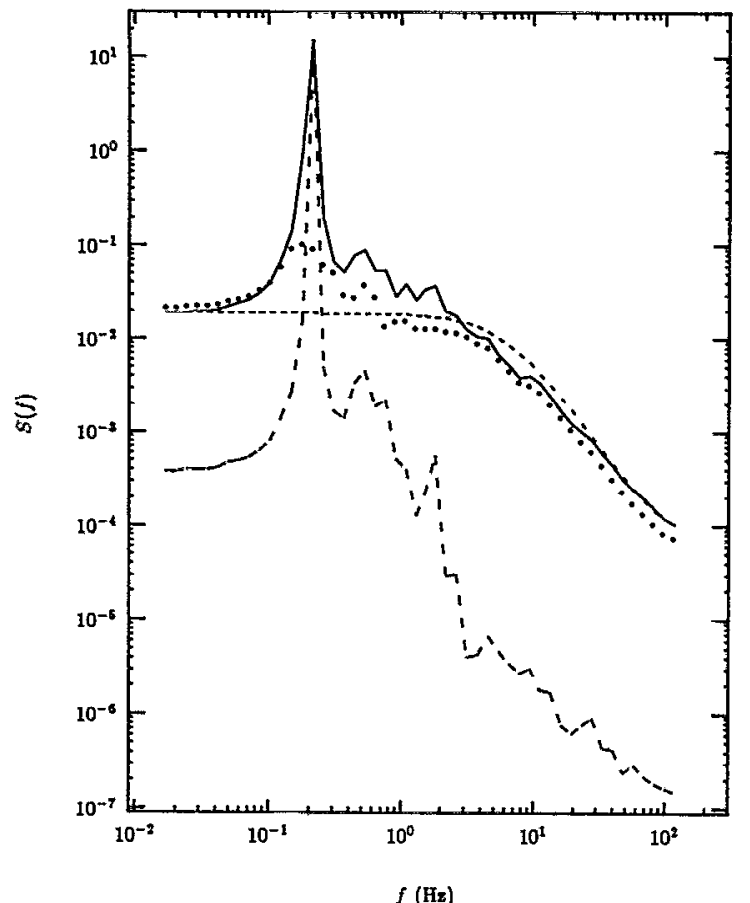

FIG. 4. Measured energy spectra of $u(t)$ and estimated energy spectra $\widetilde{u}(t)$ and $u^{\prime}(t)$ in a flat-plate boundary layer undergoing forced sinusoidal free-stream oscillation, according to the turbulence filter decomposition;-, the spectrum of $u(t) ;---$, initial estimate of the $u^{\prime}(t)$ spectrum; $\cdots$, the decomposed $u^{\prime}(t)$ spectrum;---, the decomposed $\tilde{u}(t)$ spectrum.

scales of the parent boundary layer-too rapid to allow a quasisteady response, yet too slow to "freeze" the turbulence at its mean value everywhere except near the wall ${ }^{34}$ - and so a nonstationary turbulence field was anticipated in response to the continually changing shear field. Experimental details are described by Brereton et al. ${ }^{25}$ Estimates of $S_{u u}(f), S_{\widetilde{u}_{\text {est }} \tilde{u}_{\text {est }}}(f)$, and $S_{u_{\text {est }}^{\prime} u_{\text {est }}^{\prime}}(f)$ obtained with the stationary-flow minimization approach of Sec. IV $\mathrm{A}$ are shown in Fig. 4. The cross-correlation coefficient between $\tilde{u}_{\text {est }}$ and $u_{\text {est }}^{\prime}$ achieved in this minimization was also of the order of $10^{-9}$. Of particular interest were the energy contents of the organized and turbulent components close to the forcing frequency, which may be thought of as organized and somewhat random in phase, respectively. The constraints on the smoothness of the turbulence spectrum guide the minimization toward a very realistic decomposition, compared to phase-averaged decompositions in similar flows, shown in Figs. 1 and 2.

Turbulence filter decompositions localized around individual points in a data series have also been carried out using an instantaneous power spectrum (IPS) estimation technique, with a Gaussian window chosen to attenuate information distant from the location of interest. A typical cycle decomposed with a window centered around $\omega t$ $\equiv 180^{\circ}$ is shown in Fig. 5, together with a phase-averaged estimate of the organized motion. The contamination of the phase average with information from other cycles, which do not identically reproduce the organized compo-
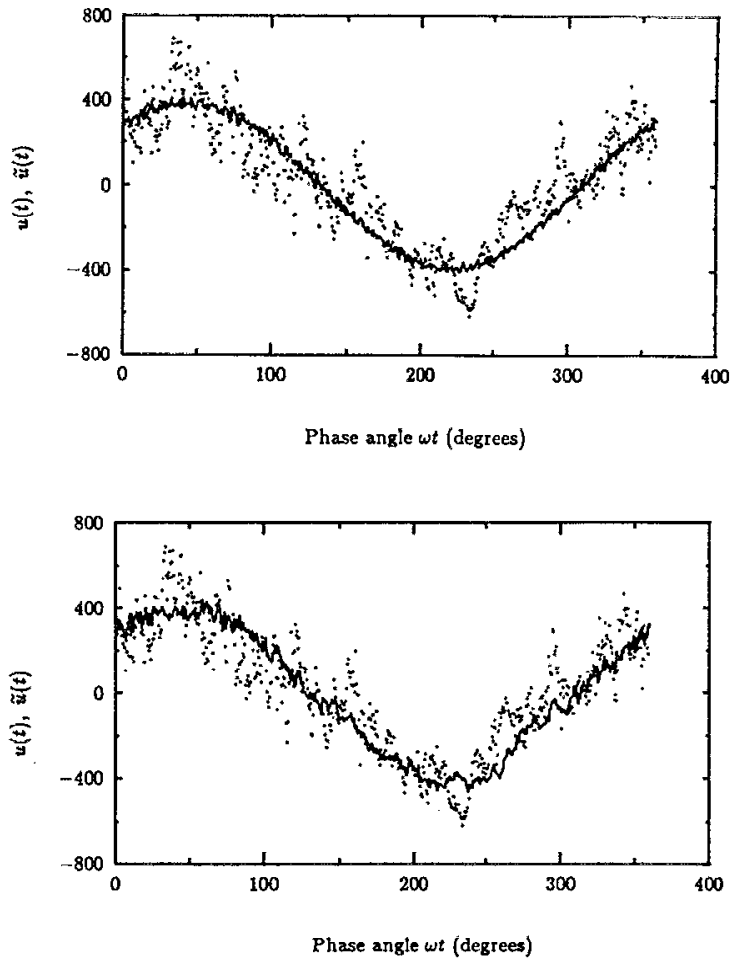

FIG. 5. A cycle of data from the time serics used for Fig. 4, together with the organized motion deduced: (i) by turbulence filter decomposition localized by an instantaneous power spectrum and a Gaussian window lapering to $1 \%$ two cycles away (in the upper plot); and (ii) by phase averaging (in the lower plot). The scaling of the ordinate is chosen for convenience.

nent of motion accounts for discrepancies. Localized time series decompositions have obvious advantages in flows with cycle-to-cycle variation in organized motion. ${ }^{20}$ They can also allow realistic estimates to be made of temporal turbulence behavior such as integral scales of time, which are rarely possible with phase-averaged decompositions. Since the turbulence filter decomposition relies only on average descriptions of the turbulence energy spectrum to establish estimates of organized and turbulent motion, no specification of a period of organized unsteadiness is necessary. The decomposition is then an attractive one for complex shear flows of a distinct spectral signature, with organized motion that cannot be specified. In such cases, the turbulent motion identified by a turbulence filter decomposition may serve as a definition for complex shearflow turbulence, in the absence of more precise information on organized motion.

While time series of streamwise velocity provide good test cases against which to qualify the turbulence filter decomposition, this perspective reveals little about the natural organization of turbulent motions. In the following section, space series of data, which reveal such motions clearly, are decomposed by the turbulence filter.

\section{APPLICATIONS TO TWO-DIMENSIONAL DATA- SPACE SERIES}

Characterization of turbulence in a space series of multiple components of velocity may be carried out using two- 


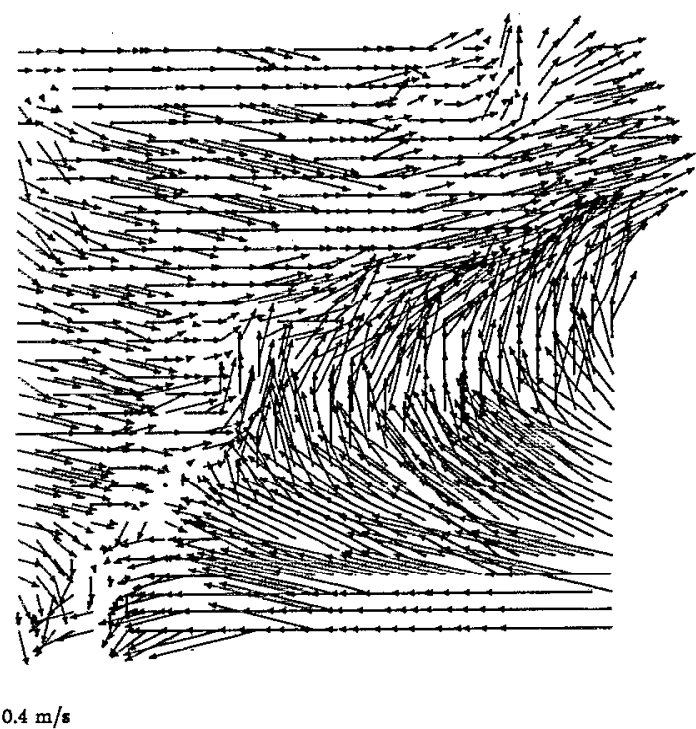

FIG. 6. Vector plot of the $u(x, y)$ and $v(x, y)$ components of the velocity field, after subtraction of their means, in a plane through the axis of a round jet $\left(\operatorname{Re}_{D}=6100\right)$, measured by particle image velocimetry. The mean velocity is $8 \mathrm{~m} / \mathrm{s}$ in the $x$ direction.

or three-dimensional turbulence filter functions: $\Phi\left(f_{\mathrm{x}}, \mathbf{x}\right)$, where $f_{\mathbf{x}}$ are the spatial frequencies in the direction of the spatial position vector $\mathbf{x}$. The data we consider were obtained from planar PIV images of the velocity field in a premixed flame of $4 \%$ propane in air, issuing as a round jet from a pipe at $\operatorname{Re}_{D}=6100$, seeded with $\mathrm{TiO}_{2}$ particles. The measurement technique entailed double pulsing a $532 \mathrm{~nm}$ beam from a Nd:YAG laser, with $20-40 \mu$ s pulse separation and a $20 \mathrm{~ns}$ individual pulse duration, to produce a laser sheet of $15 \mathrm{~mm}$ width and $300 \mu \mathrm{m}$ thickness. ${ }^{35}$ The images were interrogated following the procedure of Adrian $^{36}$ to produce planar vector fields of the kind shown in Fig. 6-a $32 \times 32$ section from the center of a $128 \times 128$ region positioned, as shown in Fig. 7. This figure shows the momentary values of velocity vectors throughout the field, after removal of their mean; they are essentially small vari-

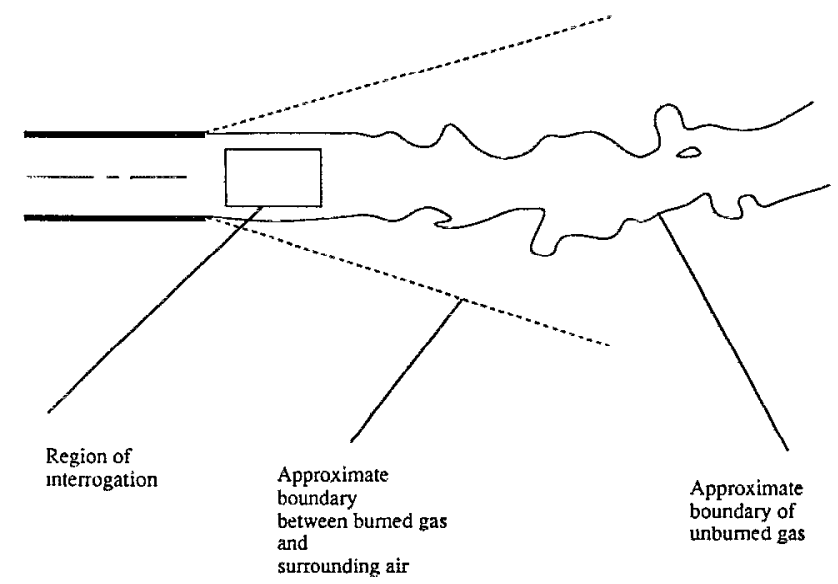

FIG. 7. Location of the interrogation region with respect to the exit plane of the jet.

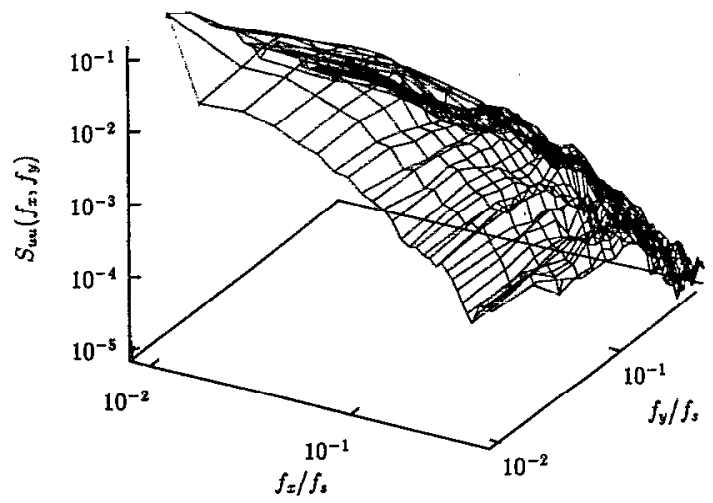

FIG. 8. Measured energy spectral density of $u(x, y)$ from the data series of Fig. 6 , after subtraction of the spatial mean.

ations about an almost uniform flow. The axis of the jet was along the center of the interrogation region, which spanned $90 \%$ of the pipe diameter and extended 1.4 diam along the axis in a region that comprised predominantly unburnt gas. Since the coherent motions of jets are known to persist for many diameters and can be captured almost in their entirety by PIV images of the lateral scale of the flow, a turbulence filter would be expected to isolate them as organized motion. Moreover, within a diameter or so of the pipe exit plane, there is little opportunity for complex interaction with other large-scale motions, and so one might expect large-scale motions to be highly coherent.

Prior to computing the minimization problem, an initial estimate of the instantaneous background turbulence field was made from the two-dimensional component energy spectra: $S_{u u}\left(f_{x}, f_{y}\right)$ and $S_{v v}\left(f_{x}, f_{y}\right)$, shown in Figs. 8 and 9. Smooth two-dimensional von Kármán models of the estimated turbulence spectra were fitted through high- and intermediate-frequency spectral densities in each of $S_{u t}\left(f_{x}, f_{y}\right)$ and $S_{v v}\left(f_{x}, f_{y}\right)$ to generate the initial condition on $S_{u^{\prime} u^{\prime}}\left(f_{x}, f_{y}\right)$ and $S_{v^{\prime} v^{\prime}}\left(f_{x}, f_{y}\right)$ for the minimization problem. The models were fitted over these regions of the frequency domain because of the smoothness of $S_{u^{\prime} u^{\prime}}\left(f_{x}, f_{y}\right)$ and $S_{v^{\prime} v^{\prime}}\left(f_{x}, f_{y}\right)$ there, and the more com-

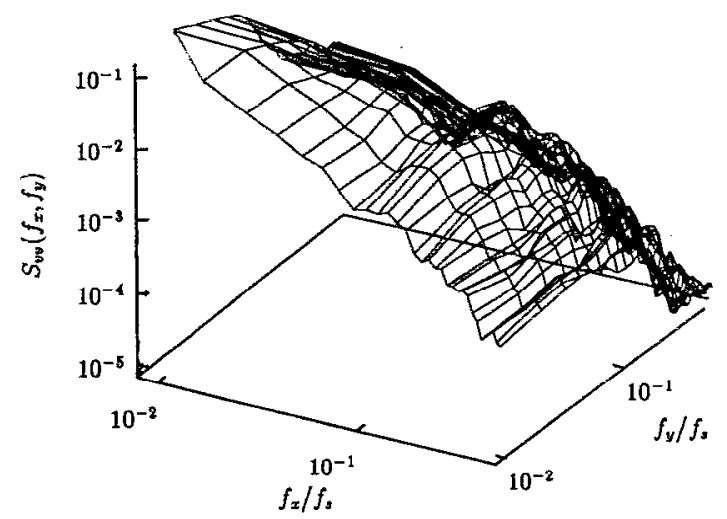

FIG. 9. Measured energy spectral density of $v(x, y)$ from the data series of Fig. 6, after subtraction of the spatial mean. 


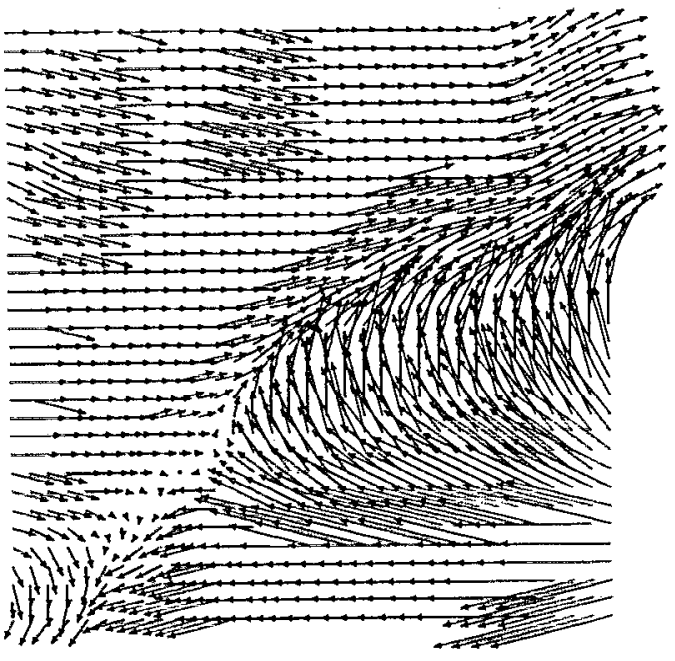

$0.4 \mathrm{~m} / \mathrm{s}$

FIG. 10. Vector plot of the $\widetilde{u}(x, y)$ and $\tilde{v}(x, y)$ components of the organized velocity field, estimated by turbulence filter decomposition.

plex topology (indicative of organized motion) at low frequencies. Orthogonality between each background turbulence and organized field was sought through iteration on $\Phi\left(f_{x}, f_{y}\right)$ to minimize the zero displacement crosscorrelation coefficient sum: $\left|R_{\bar{u}_{\text {est }} u_{\text {est }}}\right|+\left|R_{\widetilde{v}_{\text {est }} v_{\text {est }}^{\prime}}\right|$ $+\left|R_{\tilde{v}_{\text {est }} u_{\text {est }}^{\prime}}\right|+\left|R_{\tilde{u}_{\text {est }} v_{\text {est }}^{\prime}}\right|$ over the domain. Estimates of the decomposed organized and background turbulence fields from this single realization are shown in Figs. 9 and 10 for the central $32 \times 32$ region of a $64 \times 64$ field. These estimates scarcely changed during systematic variation about the initial conditions on $S_{u^{\prime} u^{\prime}}\left(f_{x}, f_{y}\right)$ and $S_{v^{\prime} v^{\prime}}\left(f_{x}, f_{y}\right)$, indicating a strong local minimum in the cross-correlation sum.

Since the natural coherent motions of the jet are more broadband in character than forced motions in the time series of Sec. V, the estimation uncertainty is greater and the orthogonality between estimates of the organized and background-turbulent fields weaker, reaching a constrained minimum with all cross-correlation coefficients less than $2 \%$. The organized field is shown in Fig. 10 (after subtraction of the spatial mean flow) and displays numerous features associated with coherent motions and organized inhomogeneous flow. Among these are the gentle curvature of the momentary pipe exit profile at the extreme left of the domain, the large-scale clockwise sweeping motion, and the saddle point toward the lower left corner of the domain (see Cantwell ${ }^{13}$ for a discussion of the significance of this feature). Decompositions of other realizations of the same flow revealed information that was characterized by visually similar features and was statistically equivalent. With the exception of edge effects, these decomposed fields showed minimal sensitivity to doubling $(128 \times 128)$ or halving $(32 \times 32)$ the size of the interrogation region and so a localized filter was not considered necessary for these data.

The companion background turbulence field is shown in Fig. 11. At first sight, it has the appearance of vector

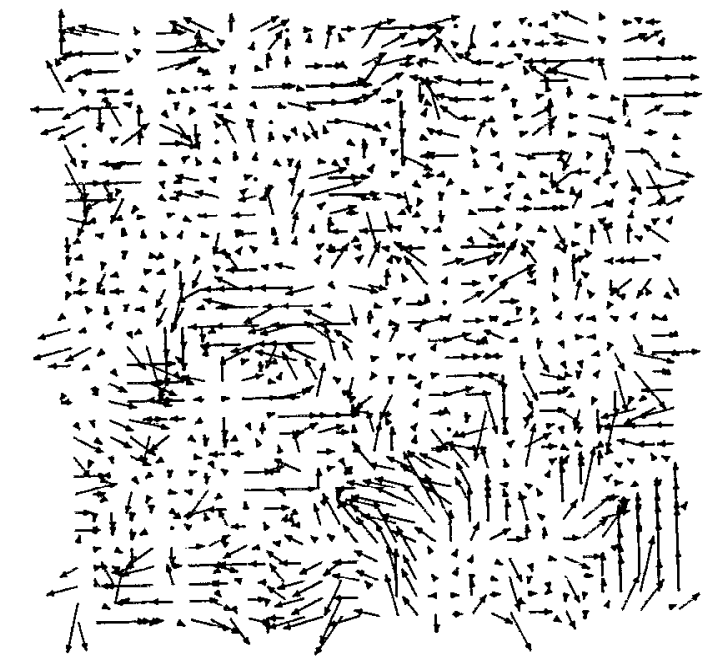

$\longrightarrow 0.4 \mathrm{~m} / \mathrm{s}$

FIG. 11. Vector plot of the $u^{\prime}(x, y)$ and $v^{\prime}(x, y)$ components of the turbulent velocity field, estimated by turbulence filter decomposition.

fields from full simulations of stationary turbulent flow (i.e., Fig. 9 in the homogeneous shear-flow simulation of Rogers and Moin $^{37}$ ). That it appears turbulent and not random or noisy may be demonstrated by computing the cross-corrclation of $u^{\prime}$ and $v^{\prime}$ within the regions of the strongest organized shear strain $\frac{1}{2}(\partial \tilde{u} / \partial y+\partial \tilde{v} / \partial x)$ at the moment of interrogation. A graph of this variation is shown in Fig. 12, in which the increasingly negative correlation in regions of increasingly organized shear is consistent with knowledge of well-understood stationary flows, when turbulence is deduced from a Reynolds decomposition. In the filter decomposition, contamination of the turbulence field with local organized motion might be expected to upset this trend, though it is not evident in these data.

A rough check on the fidelity of the decomposition was possible through knowledge of isotropic relations of turbulence scales as functions of the local turbulent Reynolds number. ${ }^{28}$ By calculating the integral scale $l$ of the decom-

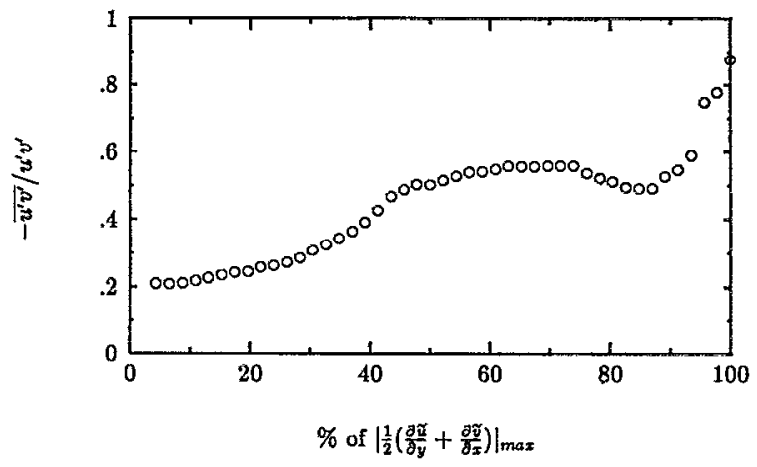

FIG. 12. Dimensionless Reynolds stress- $u^{\prime} v^{\prime}$ as a function of the relative size of the organized shear strain. Averages are conditioned on the local organized shear exceeding the abscissa value. 
posed turbulence field (taken as the average of the four almost equal integral scales of velocity) and the turbulence intensity $u^{\prime}$, the turbulent Reynolds number $\operatorname{Re}_{l}$ was estimated. The isotropic turbulence relation:

$$
\frac{\lambda}{l} \sim \sqrt{\frac{15}{\mathrm{Re}_{l}}}
$$

then leads to an estimate of the Taylor microscale $\lambda$. The reciprocal time scale $u^{\prime} / \lambda$ should be approximately equal to the RMS rate of strain of the turbulence field. Using the decomposed turbulence field of Fig. 11, the RMS rates of turbulent extensional strain: $\sqrt{\overline{\left(\partial u^{\prime} / \partial x\right)^{2}}}$ and $\sqrt{\overline{\left(\partial v^{\prime} / \partial y\right)^{2}}}$ took values of $\sim 2140 \mathrm{~s}^{-1}$, which differed from the reciprocal turbulent time scale $\left(u^{\prime} / \lambda \sim 2360 \mathrm{~s}^{-1}\right)$ by only about $10 \%$. The comparability of these estimates is thought to be as good as the validity of the isotropic relations used to form them, which are believed to scale in proportion to a constant of order unity. This result is noteworthy because $a d$ hoc techniques for interrogating the turbulent structure of PIV fields, such as Gaussian spatial filtering with arbitrarily determined widths, might well recover organized velocity fields similar to those in Fig. 10. However, they are known to yield artificial integral scales dependent on filter width, and are unlikely to simultaneously recover background turbulence fields with statistics comparable to those of Fig. $11 .^{35}$

The streamwise velocity derivative (before decomposition) had a negative skewness, defined as

$$
S=\frac{-\overline{\left(\partial u^{\prime} / \partial x\right)^{3}}}{\left[\overline{\left(\partial u^{\prime} / \partial x\right)^{2}}\right]^{3 / 2}},
$$

which took a value of 0.5 , consistent with many other developed turbulent flows over a wide range of turbulent Reynolds numbers. ${ }^{38}$ When the velocity derivative skewness of the decomposed organized and backgroundturbulence components were averaged in regions that did not suffer from edge effects, negative skewnesses $(S \simeq 0.5)$ were also found. Since negative skewness is associated with the production of mean-square vorticity through vortex stretching, ${ }^{39}$ this result implied that production of meansquare vorticity was significant in both organized and background-turbulent motions of the jet. The most orderly momentary correlation found between the momentary coherent field and structural features of the background turbulence field was a gentle trend toward more negative skewness in the velocity derivative of the streamwise background turbulence, in regions in which the coherent velocity vector was more normally aligned to the jet axis. This variation of $-\overline{\left(\partial u_{\text {est }}^{\prime} / \partial x\right)^{3}} /\left[\overline{\left(\partial u_{\text {est }}^{\prime} / \partial x\right)^{2}}\right]^{3 / 2}$ with $|\widetilde{v}| / \sqrt{\widetilde{u}^{2}+\widetilde{v}^{2}}$ is shown in Fig. 13. The weak dependence of $S$ on the alignment of the momentary coherent velocity vector is synonymous with the excellent collapse of this parameter with turbulent Reynolds number, regardless of whether it is in duct flows, atmospheric flows, or isotropic or homogeneous flows, ${ }^{38}$ all of which feature very different coherent motions.

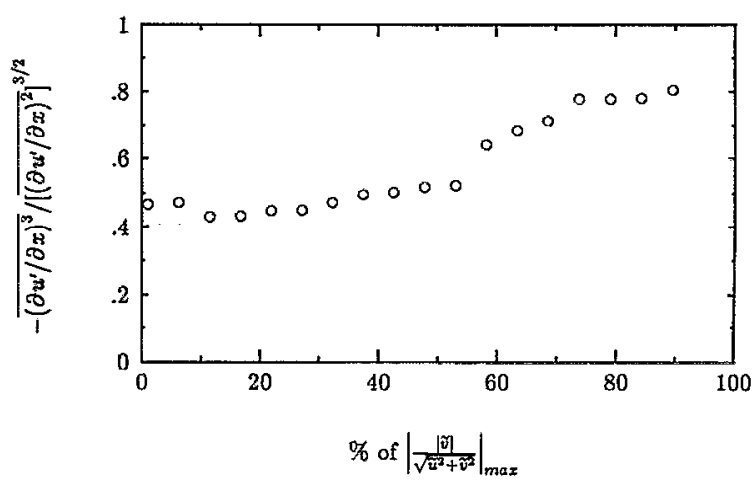

FIG. 13. Velocity derivative skewness as a function of the relative magnitude of the organized velocity vector, normal to the jet axis. Averages are conditioned on the local organized velocity vector exceeding the $a b$ scissa value.

These findings are significant insofar as they illustrate that the turbulence filter decomposition is one that distinguishes between organized and background turbulent motions in a physically appealing way. They also allow the momentary coupling between organized and more random motion to be examined for the purposes of characterizing turbulence. Temporal sequences of such realizations allow study of the evolution of component field interactions, which would then offer an experimental means of examining how rcalistically structural models of background turbulence might be related to large-scale coherent motions.

\section{DISCUSSION AND CONCLUDING REMARKS}

A turbulence filter has been demonstrated for firstorder estimations of organized fluid motion, as revealed by the energy spectra of data series from turbulent shear flows. As a decomposition procedure, it has the physical appeal of describing turbulence as the sum of its more organized and its background motions, allowing examination of these individual decomposed fields and their coupling.

The effectiveness of any technique for identifying organized motion depends on the information available. While single-point time series can reveal little but bulk organized unsteadiness, space series of turbulent data yield realistic decompositions of both organized and turbulent motion, whose coupling and statistics appear to be quite plausible. While these decompositions have achieved reasonable results with the simpler real filter, a fully complex implementation offers potentially greater accuracy, at the cost of providing information on the cross-spectrum of organized and background-turbulent components. Tuning of this kind would require knowledge of specific organized motions, in particular turbulent lows, though it might offer significant improvement when there is broadband overlapping of organized and turbulent energy spectra. A complex filter could also be more effective in identifying particular kinds of organization for which it might be tuned, in single-point time series. Other restrictions on filter performance include nonstationarities that are too 
severe for time/space-frequency techniques. Each type of nonstationary behavior must be treated as an individual case, and it remains to be established whether there are recognizable subclasses of nonstationarity in turbulent flows. It also remains to be demonstrated from first principles that organized motions naturally make the shapes of spectra dissimilar to the smoothly varying ones expected for background turbulent motions. This challenge may amount to finding a formal field decomposition, for each component of which the desired spectral properties may be demonstrated rigorously from solutions of Navier-Stokes equations.

Extension of the decomposition procedure to threedimensional flows is straightforward, notwithstanding the increased dimensionality of the minimization problem. In the case of incompressible flow, the orthogonal decomposed fields must also satisfy a continuity equation; a summation of $\left|\partial \tilde{u}_{i} / \partial x_{i}\right|+\left|\partial u_{i}^{\prime} / \partial x_{i}\right|$ over the domain may be added to the cross-correlation sum to be minimized. The decomposition can be applied to scalar fields or fields of any other turbulent tensor quantity, provided the energy spectrum of the turbulent part of that measure has an identifiable shape, which distinguishes it from organized contributions. For this reason it is particularly well suited to the velocity field in well-developed turbulent shear flows with organized temporal or spatial complexity.

The ability of this decomposition to naturally separate shear-flow turbulence into organized and more random components has some interesting implications for the modeling of turbulence. Rather than trying to devise models for unresolved scales, as in large eddy simulations, it suggests modeling of all background turbulent motions through their interaction with large-scale coherent motions, shifting the modeling emphasis to the interaction between coherent and background turbulent motion. These ideas share similarities with those of Townsend ${ }^{40}$ on shear-flow turbulence, as comprising large eddies that respond to the organized strain of the mean flow and smaller eddies that are strained more randomly and form a roughly isotropic background turbulence field. The turbulence filter decomposition also shows promise for applications in complex flows in which the organized motions are not sufficiently predictable to allow other averaging techniques to be used. In such cases, this characterization can provide a definition of turbulence, as the orthogonal partner to organized motion, with an energy spectrum of comparable shape to its stationary-flow counterpart. As such, it represents a useful new approach to understanding the complexities of turbulent shear flow.

\section{ACKNOWLEDGMENTS}

The authors gratefully acknowledge the assistance of D. Reuss in providing the PIV velocity data series. The second author acknowledges the support of the Ministry of Education, Turkey.

\footnotetext{
'O. Reynolds, "On the dynamical theory of incompressible viscous fluids and the determination of the criterion," Philos. Trans. R. Soc. London Ser. A 186, 123 (1895).
}

${ }^{2}$ O. M. Phillips, The Dynamics of the Upper Ocean (Cambridge University Press, Cambridge, 1967).

${ }^{3}$ R. T. Beyer, Nonlinear Acoustics (Naval Sea System Command, Washington, DC, 1974).

${ }^{4}$ A. K. M. F. Hussein and W. C. Reynolds, "The mechanics of an organized wave in turbulent shear flow," J. Fluid Mech. 41, 241 (1970).

${ }^{5}$ W. W. Willmarth and S. S. Lu, "Structure of the Reynolds stress near the wall," J. Fluid Mech. 55, 481 (1971).

${ }^{6} \mathrm{H}$. P. Bakewell and J. L. Lumley, "Viscous sublayer and adjacent wall region in turbulent pipe flow," Phys. Fluids 10, 1880 (1967).

${ }^{7} \mathrm{M}$. N. Glauser and W. K. George, "An orthogonal decomposition of the axisymmetric jet mixing layer utilizing cross-wire measurements," Turbulent Shear Flows 6 (Springer-Verlag, Berlin, 1987).

${ }^{8} \mathrm{P}$. Moin and R. D. Moser, "Characteristic eddy decomposition of turbulence in a channel," J. Fluid Mech. 200, 471 (1989).

${ }^{9}$ R. J. Adrian and P. Moin, "Stochastic estimation of organized turbulent structure: homogeneous shear flow," J. Fluid Mech. 190, 531 (1988).

${ }^{10}$ A. K. M. F. Hussain, "Coherent structures-Reality and myth," Phys. Fluids, 26, 2816 (1983).

${ }^{11}$ L. Zubair, K. R. Sreenivasan, and M. V. Wickerhauser "Characterization and compression of turbulent signals and images using waveletpackets," in Studies in Turbulence, edited by T. B. Gatski, S. Sarkar, and C. G. Speziale (Springer-Verlag, Berlin, 1992), p. 489.

${ }^{12} \mathrm{M}$. Farge, "Wavelet transforms and their applications to turbulence," Annu. Rev. Fluid Mech. 24, 395 (1992).

${ }^{13}$ B. J. Cantwell, "Organized motion in turbulent flow," Annu. Rey. Fluid Mech. 13, 457 (1981).

${ }^{14} \mathrm{~W}$. W. Willmarth, "Structure of turbulence in boundary layers," Adv. Appl. Mech. 15, 159 (1975).

${ }^{15}$ R. A. Antonia, "Organized motion in a turbulent boundary layer," Proceedings of the 7th Australasian Hydraulics and Fluid Mechanics Conference, 1980, p. 155.

${ }^{16} \mathrm{~J}$. M. Wallace, "Observations on the nature and mechanism of the bounded turbulent shear flow structure," Structure and Chaotic Phenomena in Fluids, edited by T. Tatsumi (North-Holland, Amsterdam, 1984), p. 477.

${ }^{17}$ A. K. M. F. Hussain, "Coherent structures and turbulence," J. Fluid Mech. 173, 303 (1986).

${ }^{18}$ J. L. Lumley, Stochastic Tools in Turbulence (Academic, New York, 1970), p. 80.

${ }^{19}$ P. Z. Peebles, Probability, Random Variables and Random Signal Principles (McGraw-Hill, New York, 1980), p. 265.

${ }^{20} \mathrm{G}$. J. Brereton and A. Kodal, "A frequency-domain filtering technique for triple decomposition of unsteady turbulent flow," J. Fluids Eng. 114, 1 (1992).

${ }^{21}$ J. C. R. Hunt and D. J. Carruthers, "Rapid distortion theory and the 'problems' of turbulence," J. Fluid Mech. 212, 497 (1990).

${ }^{22}$ G. E. P. Box and G. M. Jenkins, Time Series Analysis: Forecasting and Control (Holden-Day, Oakland, CA, 1976).

${ }^{23}$ W. C. Reynolds, "Computation of turbulent flows," Annu. Rev. Fluid Mech. 8, 183 (1976).

${ }^{24}$ R. S. Rogallo, "Numerical experiments in homogeneous turbulence," NASA Tech. Memo. TM 81315 (1981).

${ }^{25}$ A. E. Perry, S. Henbest, and M. S. Chong, "A theoretical and experimental study of wall turbulence," J. Fluid Mech. 165, 163 (1986).

${ }^{26}$ G. J. Brereton and W. C. Reynolds, "Dynamic response of boundary layer turbulence to oscillatory shear," Phys. Fluids A 3, 178 (1991).

${ }^{27}$ J.-L. Hwang, "An experimental study of the fluid mechanics of turbulent pipe flow when subjected to forced oscillation at high frequencies," Ph.D. thesis, The University of Michigan, Ann Arbor, MI, 1992.

${ }^{28}$ N. Aubry, R. Guyonnet, and R. Lima, "Turbulence spectra," J. Stat. Phys. 67, 203 (1992).

${ }^{29}$ N. Aubry, R. Guyonnet, and R. Lima, "Role of the dilation symmetry in turbulence," Proceedings of the International Conference on Wavelets and Applications, Toulouse, France, 1992.

${ }^{30} \mathrm{H}$. Tennekes and J. L. Lumley, A First Course in Turbulence (MIT Press, Cambridge, MA, 1972), p. 260.

${ }^{31}$ R. D. Hippensteil and P. M. de Oliveira, "Time-varying spectral estimation using the instantaneous power spectrum (IPS)," IEEE Trans. Acoust. Speech Signal Proc. ASSP-38, 10 (1990).

${ }^{32}$ G. J. Brereton, W. C. Reynolds, and R. Jayaraman, "Response of a turbulent boundary layer to sinusoidal free-stream unsteadiness," $\mathrm{J}$. Fluid Mech. 221, 131 (1990).

${ }^{33}$ S. J. Kline, W. C. Reynolds, F. A. Schraub, and P. W. Runstadler, "The 
structure of turbulent boundary layers," J. Fluid Mech. 30, 741 (1967).

${ }^{34}$ B. R. Ramaprian and S. W. Tu, "Fully developed periodic turbulent pipe flow. Part 2. The detailed structure of the flow," J. Fluid Mech. 137, 59 (1983).

${ }^{35}$ D. L. Reuss, R. J. Adrian, C. C. Landreth, D. T. French, and T. D. Fansler, "Instantaneous planar measurements of velocity and largescale vorticity and strain rate in an engine using particle-image velocimetry," SAE Paper 890616, 1989.

${ }^{36} \mathrm{R}$. J. Adrian, "Statistical properties of particle image velocimetry measurements in a turbulent flow," Laser Anemometry in Fluid Mechanics-III, edited by R. J. Adrian, T. Asanuma, D. F. G. Durao,
F. Durst, and J. H. Whitelaw (Ladoan, Lisbon, 1988).

${ }^{37}$ M. M. Rogers and P. Moin, "Structure of the vorticity field in homogeneous turbulent flows," J. Fluid Mech. 176, 33 (1987).

${ }^{38} \mathrm{~S}$. Tavoularis, J. C. Bennett, and S. Corrsin, "Velocity-derivative skewness in small Reynolds number, nearly isotropic turbulence," J. Fluid Mech. 88, 63 (1978).

${ }^{39} \mathrm{G}$. I. Taylor, "Production and dissipation of vorticity in a turbulent fuid," Proc. R. Soc. London Ser. A 164, 15 (1938).

${ }^{40} \mathrm{~A}$. A. Townsend, "The response of sheared turbulence to additional distortion," J. Fluid Mech. 81, 171 (1980). 